\title{
Fabrication of redispersible silica nanoparticles by a facile one-step one-pot approach
}

\author{
Haitao Liu ${ }^{1,2}$, Hailin $\mathrm{Cao}^{3, *}$, Yudong Huang ${ }^{1, * *}$, Pengcong Shao ${ }^{2}$, and Yiwu $\mathrm{Yan}^{4}$ \\ ${ }^{1}$ School of Chemistry and Chemical Engineering, Harbin Institute of Technology, Harbin 150001, China \\ ${ }^{2}$ Shenzhen Academy of Aerospace Technology, Shenzhen 518057, China \\ ${ }^{3}$ School of Materials Science and Engineering, Harbin Institute of Technology, Shenzhen 518055, China \\ ${ }^{4}$ Guangdong Advanced Polymer Composite Engineering Technology Research Center, Shenzhen 518057, China
}

\begin{abstract}
In this study, special redispersible silica nanoparticles (SNP) was synthesized by a facile one-step one-pot approach. The synthesized amorphous SNP had a spherical and uniform particle morphology with a diameter of $30 \mathrm{~nm}$ and exhibited high monodispersity. The characteristics of the particles were investigated by fourier transform infrared spectroscopy and thermogravimetric analysis. The results revealed that there were a large number of methyl groups grafted on the surface of the SNP, and the organic materials content in the as-synthesized SNP was approximately $10 \%$. After a simple ultrasonic process, the SNP solid powder showed excellent stable and transparent redispersion in various organic solvents. In addition, the results revealed that the different polarities of the solvents had a significant effect on the visible light transparency of the SNP redispersion. SNP showed the best redispersion performance in N-methyl-pyrrolidone, exhibiting a transmittance of more than $80 \%$ at $600 \mathrm{~nm}$ at a high concentration of $25 \mathrm{wt} \%$. The synthesis process developed in this study is simple, inexpensive, and can be used in the industrial application of SNP.
\end{abstract}

\section{Introduction}

In recent decades, nanotechnology has attracted significant scientific interest owing to the promising potential applications of nanoparticles and nanotechnology in various fields. ${ }^{[1-2]}$ Silica nanoparticles (SNP) such as precipitated silica, fumed silica, and colloidal silica are inorganic chemical materials with an average particle size of less than $100 \mathrm{~nm}$ and are widely used in various field including the fields of functional coatings, catalysis, nanocomposites, adsorption, biomedicine, etc. ${ }^{[3-7]}$ SNP is usually added to solvents, polymers, and other matrices as special fillers in the preparation processes of functional coatings or nanocomposites. However, the agglomeration of SNP in the matrix due to its small particle size and high surface energy limits its further application. The agglomeration of SNP can be attributed to its poor dispersion property in the matrix, which in turn leads to the degradation of the physical and chemical properties of the matrix, such as a reduction in the transparency and strength of resin. In summary, the particle agglomeration caused by the poor dispersion ability of SNP significantly reduces the functional effect of its application. Consequently, several surface modification steps have been introduced to improve the dispersibility of synthesized SNP. ${ }^{[8-10]}$ However, the high cost of these surface modification steps and their inability to fundamentally improve the dispersion of SNP in the application matrix has limited the effective application of these methods. Generally, the modification of SNP with a suitable modifier and modification process improves the dispersibility of SNP in specific solvents or polymers. However, agglomeration occurs when SNP is redispersed in another solvent or polymer matrix. In addition, due to the extremely high surface activity of SNP, synthesized or modified SNP are prone to secondary agglomeration during the drying process, which reduces the redispersibilities of dried SNP in solvents or polymers. ${ }^{[11-12]}$ SNP with good redispersibility can be packaged as solid powders to avoid storage and transportation safety problems caused by packaging the SNP in the form of dispersion in solvent. SNP solid powders with good redispersibility can be directly redispersed in different solvents or polymer matrices for practical applications, thus making them more flexible and easy to use. However, studies on the redispersibility of SNP and the preparation of redispersible SNP are rare.

In this study, we report a facile approach to fabricate redispersible SNP by a one-step one-pot method without additional surface modification treatment. The dried SNP solid powder showed excellent redispersible properties and was easily redispersed in various organic solvents to obtain highly stable and transparent SNP redispersion.

\section{Experiment}

\subsection{Materials}

Corresponding author: **huangyd@hit.edu.cn; *caohl@hit.edu.cn; 
Tetraethoxysilane (TEOS, AR), ethanol (ETOH, AR), tetrahydrofuran (THF, AR), ethyl acetate (EA, AR), N-methyl-pyrrolidone (NMP, AR), and ammonium hydroxide (NH4OH, 25-28\%, AR) were purchased from Damao Chemical Reagent Factory (Tianjin, China). Dimethoxydimethylsilane (DDS, AR) was purchased from Hangzhou Jessica Chemical Co., Ltd (Hangzhou, China). The commercial SNP powder was purchased from Wacker Chemic Co. Ltd (Germany). All chemicals were used as received without any further purification.

\subsection{Synthesis of the redispersible SNP}

The redispersible SNP was synthesized by a one-step one-pot method based on a modified Stöber protocol.[13] To explain in brief, ETOH $(150 \mathrm{~g})$, NH4OH $(10 \mathrm{~g})$, TEOS (10 g), and DDS (2.88 g) were placed in a three-neck round-bottom flask, after which the mixture was stirred vigorously at $38^{\circ} \mathrm{C}$ for $6 \mathrm{~h}$. After the reaction, the redispersible SNP dispersion in ETOH was obtained. After drying the dispersion in an oven at $60{ }^{\circ} \mathrm{C}$ for $4 \mathrm{~h}$, the redispersible SNP solid powder was obtained.

\subsection{Characterization}

X-ray powder diffraction (XRD) analysis of the as-synthesized SNP was conducted (DX-2700BH,
Haoyuan). The chemical structure of the as-synthesized SNP was examined using a Fourier transform infrared (FTIR) spectrometer (TENSORII, Bruker). Transmission electron microscopy (TEM, F30, FEI Tecnai) was conducted to analyze the morphology of the as-synthesized SNP. The thermal stability of the as-synthesized SNP was determined through thermogravimetric analysis (TGA, STA 449 F5, Netzsch). The optical transparency of the SNP dispersions was examined by UV-visible (UV-vis) spectroscopy (UV-1800, Shimadzu).

\section{Results and discussion}

\subsection{Characterization of the as-synthesized SNP}

The XRD pattern of the as-synthesized SNP is shown in Figure 1. The XRD diffraction pattern reveals that the synthesized SNP possessed an amorphous-like structure. TEM was carried out to investigate the morphology and the size of the SNP, and the TEM images are shown in Figure 2. The high- and low-magnification TEM images reveal that the well-dispersed SNP had a spherical and uniform particle morphology with a diameter of approximately $30 \mathrm{~nm}$.

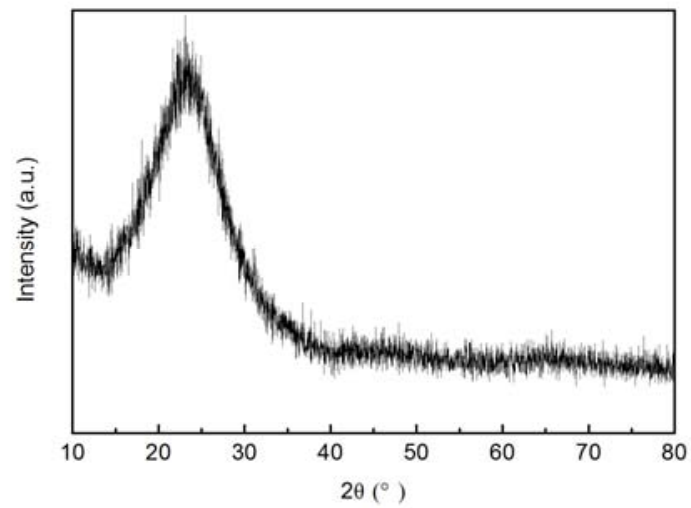

Figure 1. XRD pattern of the as-synthesized SNP.
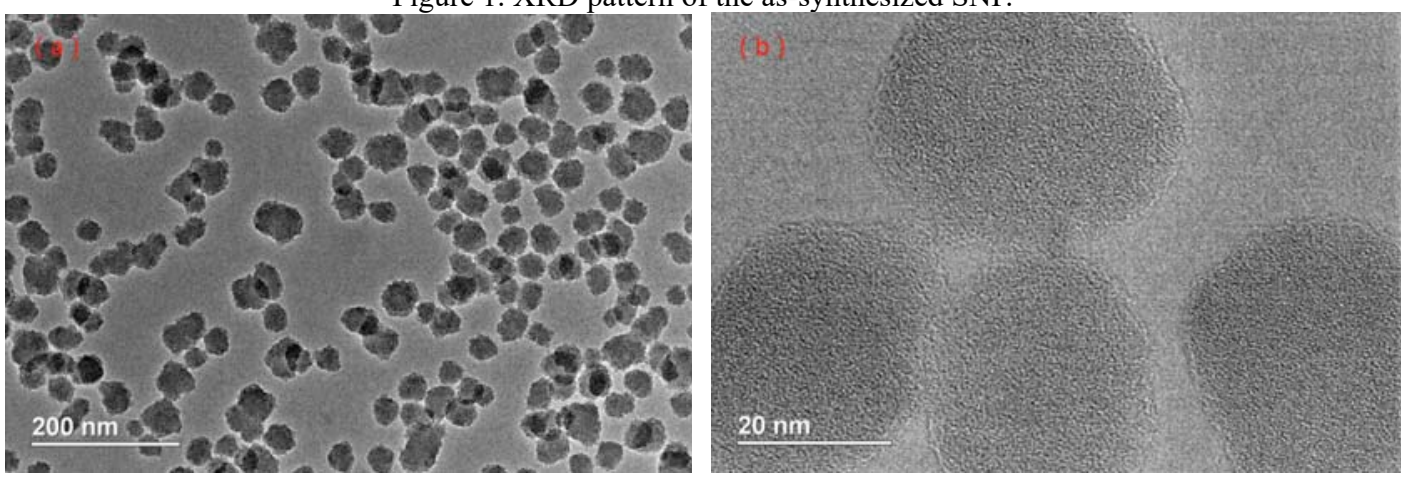

Figure 2. TEM images of the as-synthesized SNP at (a) low and (b) high magnifications.

Figure 3 shows the FTIR spectra of the as-synthesized SNP. As seen, an absorption band appeared at approximately $1095 \mathrm{~cm}-1$, which could be attributed to the vibrations of Si-O-Si bonds.[14] Additional absorption peaks were observed at $\sim 2865$ $\mathrm{cm}-1$ and $\sim 1460 \mathrm{~cm}-1$, which could be attributed to the presence of -CH3 group.[15] The absorption peaks observed at $\sim 860 \mathrm{~cm}-1$ corresponded to the characteristic vibrations of the $\mathrm{Si}-\mathrm{CH} 3$ bonds.[16] The observed characteristic bands of the $-\mathrm{CH} 3$ and $\mathrm{Si}-\mathrm{CH} 3$ groups indicated the successful synthesis of the SNP grafted with a large number of methyl groups. Figure 4 shows the TG curve of the as-synthesized SNP. The TG curve revealed that the organic matter content of the SNP was about 10 
wt $\%$, and was completely decomposed at approximately $600{ }^{\circ} \mathrm{C}$

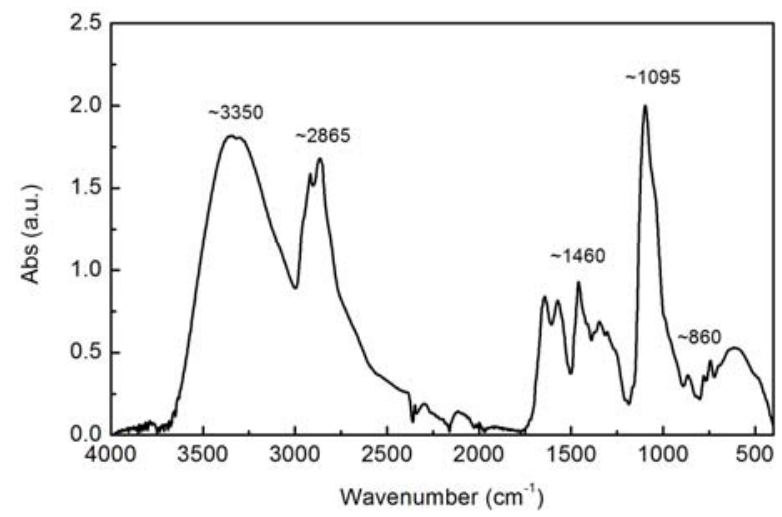

Figure 3. FTIR spectra of the as-synthesized SNP.

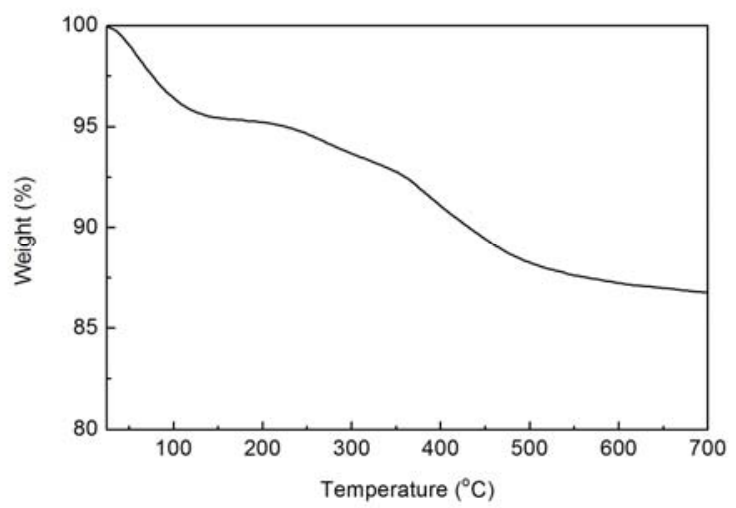

Figure 4. TGA curve of the as-synthesized SNP.

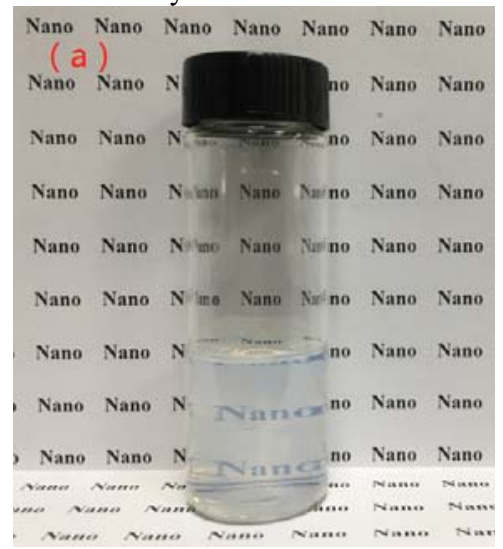

\subsection{Redispersible as-synthesized SNP}

properties

of

the

The obtained SNP was stably dispersed in ETOH, and a light-blue transparent dispersion was observed, as shown in Figure 5 (a), indicating that the SNP dispersion in ETOH exhibited excellent transparency. After drying the SNP dispersion in ETOH in an oven at $60{ }^{\circ} \mathrm{C}$ for $4 \mathrm{~h}$, a white SNP solid powder was obtained, as shown in Figure 5 (b). To investigate the redispersibility of the as-synthesized SNP, $1 \mathrm{wt} \%$ SNP content of different SNP redispersion was prepared by mixing $0.1 \mathrm{~g}$ of the dried SNP solid powder with $9.9 \mathrm{~g}$ of various solvents, after which ultrasonication was performed for $1 \mathrm{~min}$ to redisperse the SNP in the solvent. For the redispersibility investigation, we selected a variety of solvents that are widely used in industries including alcohol solvents (ETOH), ether solvents (THF), ester solvents (EA), and ketone solvents (NMP). As shown in Figure 6(a), after the simple ultrasonic dispersion process, transparent SNP redispersion was obtained in all solvents, indicating that the as-synthesized SNP exhibited excellent redispersibilities in various solvents. For comparison, the redispersibility of a commercial SNP powder in ETOH with a concentration of $1 \mathrm{wt} \%$ was investigated. As shown in the photo of the suspension in Figure 6(b), the transparency of the suspension was very low, indicating that the poor redispersibility of the commercial SNP.

Figure 5. Photos of (a) as-synthesized SNP dispersion in ethanol (ETOH), and (b) SNP solid powder after drying.
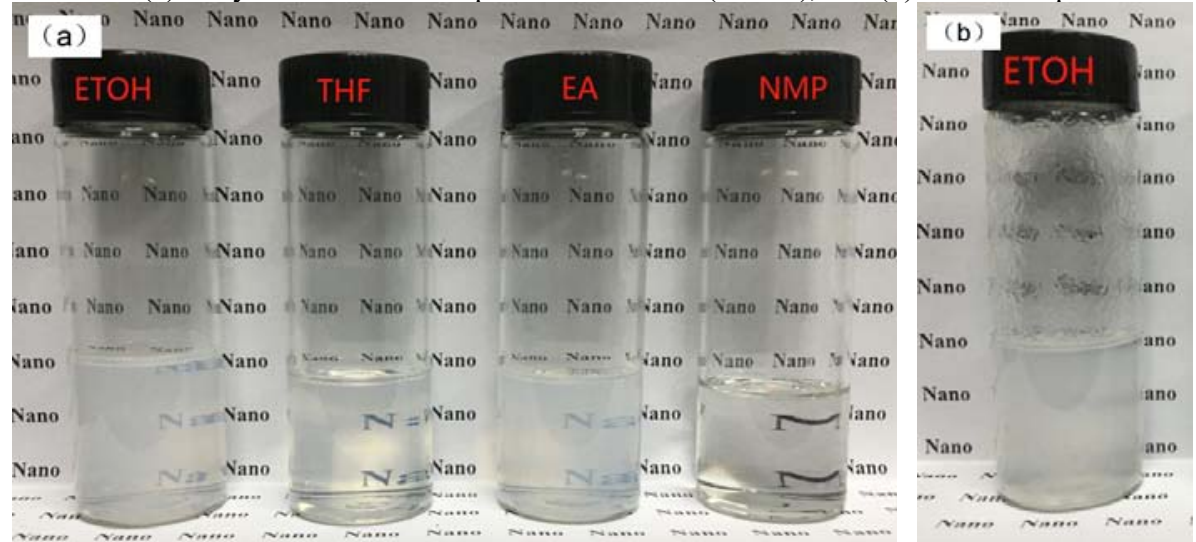

Figure 6. Photos of (a) as-synthesized SNP redispersion in different solvents, and (b) purchased SNP redispersion in ETOH. 
Figure 7 shows the UV-vis transmittance spectra of the as-synthesized SNP redispersion in different solvents and that of the purchased SNP redispersion in ETOH. The concentration of SNP in all the redispersion was 1 $\mathrm{wt} \%$. The light transmittance of the different redispersion were tested, and the results revealed that the SNP redispersion in NMP showed the highest transmittance with the light transmittance at $600 \mathrm{~nm}$ exceeding $90 \%$, whereas, the transparency of the commercial SNP redispersion in ETOH was significantly lower. In addition, there were obvious differences in the transparencies of the SNP redispersion in different solvents, indicating that the SNP has different redispersibilities in different solvents. Previous studies have reported that nanoparticles aggregates in a dispersion medium significantly increase the intensity of scattered light as described by Rayleigh's law, which would in turn induce a considerable decrease in the transparency of the dispersion.[17] Owing to the different polarities of different solvents, which affects the aggregation state of SNP in the solvent, the degrees of light scattering of the redispersion differ, which in turn affected the transparency of the redispersion. Generally, the redispersion obtained by redispersing the as-synthesized SNP solid powder in different solvents have high visible light transparency, which is consistent with the results in Figure 6, and the light transmittance of all the redispersion at $600 \mathrm{~nm}$ exceeded $50 \%$. In contrast, the light transmittance of the purchased SNP redispersed in ETOH was significantly low, and that of the suspension at $600 \mathrm{~nm}$ was less than $30 \%$, indicating the low redispersibility of the purchased SNP in ETOH. It is worth noting that the redispersion of SNP obtained by redispersing the SNP solid powder in different solvents showed excellent stabilities. After storage at room temperature for 7 days, no delamination or precipitation occurred in the transparent SNP redispersion.

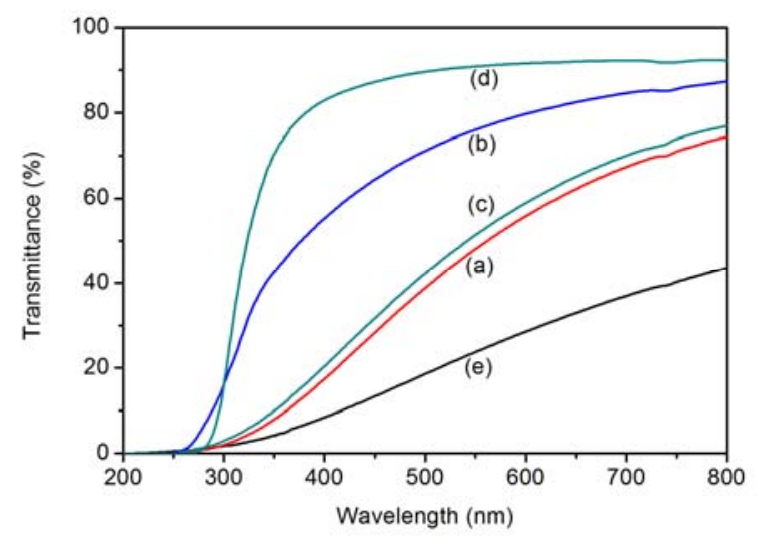

Figure 7. UV-vis transmittance spectra of as-synthesized SNP redispersion in (a) ETOH; (b) THF; (c) EA; (d) NMP, and (e) the purchased SNP redispersion in ETOH.

The results of the above analysis indicated that the as-synthesized SNP showed the best redispersibility in NMP. Therefore, we investigated the redispersibilities of different concentration of the as-synthesized SNP in NMP using a simple ultrasonic method. The photos of the redispersion are shown in the Figure 8. As seen, the redispersion with SNP concentrations of $1 \mathrm{wt} \%$ and 10 $\mathrm{wt} \%$ exhibited excellent transparencies, whereas the redispersion with $25 \mathrm{wt} \% \mathrm{SNP}$ concentration exhibited a slightly poorer transparency. The UV-vis transmittance spectra of the as-synthesized SNP redispersion in NMP at different concentrations is shown in Figure 9. The transmittance of the redispersion with $1 \mathrm{wt} \%, 10 \mathrm{wt} \%$, and $25 \mathrm{wt} \%$ SNP content at $600 \mathrm{~nm}$ was $91.6 \%, 88.1 \%$, and $83.1 \%$, whereas the transmittance of pure NMP at $600 \mathrm{~nm}$ was $92.3 \%$. This result indicated that the transmittance of the redispersion decreased gradually with an increase in the SNP content, however, they were all higher than $80 \%$, and high transparencies were maintained.

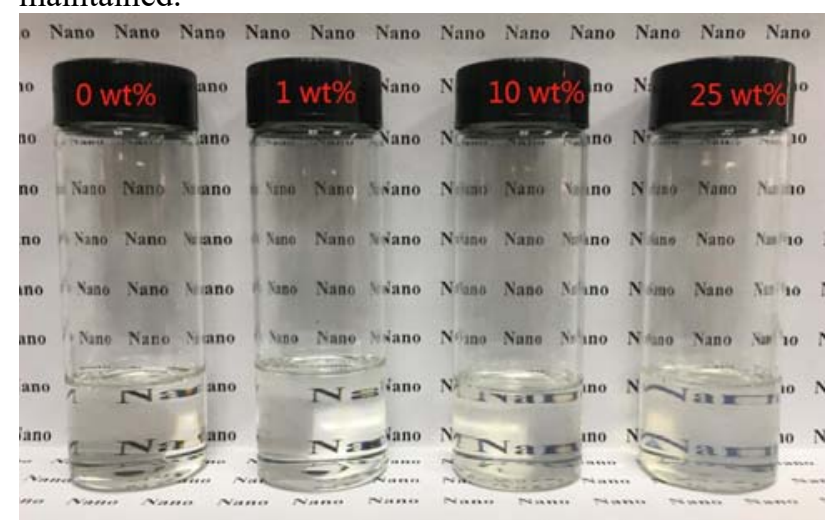

Figure 8. Photos of the as-synthesized SNP redispersed in NMP at different concentrations.

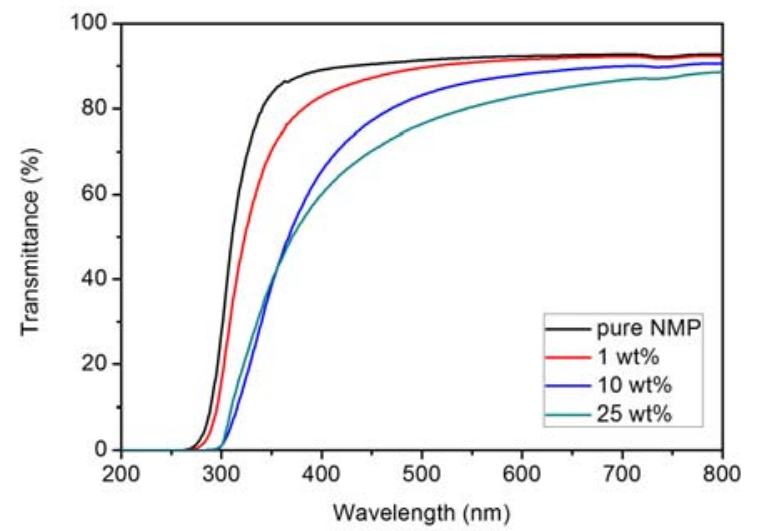

Figure 9. UV-vis transmittance spectra of the as-synthesized SNP redispersion in NMP at different concentrations.

\section{Conclusion}

In this study, we reported the synthesis of SNP by a simple one-step one-pot method. The obtained SNP with a diameter of approximately $30 \mathrm{~nm}$ exhibited a stable dispersion in ETOH. By drying the dispersion in a traditional oven, a redispersible and user-friendly SNP solid powder was obtained. The dried SNP solid powder exhibited stable redispersion in various organic solvents including ETOH, THF, EA, and NMP. In addition, the SNP powder exhibited excellent redispersion in NMP even at a high concentration of $25 \mathrm{wt} \%$. The preparation method proposed in this study can provide technical insights for the development of new SNP-based functional coatings and nanocomposites. 


\section{References}

1. X Wang, J Zhang, Q Peng, YD Li, A general strategy for nanocrystal synthesis, Nature, 2005, 437: $121-124$

2. J Loste, JM Lopez-Cuest, L Billon, H Garay. Transparent polymer nanocomposites: An overview on their synthesis and advanced properties. Progress in Polymer Science, 2019, 89: 133-158

3. LP Singh, SK Bhattacharyya, S Ahalawat, R Kumar, G Mishra, U Sharma, G Singh. Sol-gel processing of silica nanoparticles and their applications. Advances in Colloid and Interface Science, 2014, 214: 17-37

4. C Zhou, S Xu, P Pi, J Cheng, L Wang, J Yang, X Wen. Polyacrylate/silica nanoparticles hybrid emulsion coating with high silica content for high hardness and dry-wear-resistant. Progress in Organic Coatings, 2018, 121: 30-37

5. X Yang, D Chen, S Liao, H Song, Y Li, Z Fu, Y Su. High-performance $\mathrm{Pd}-\mathrm{Au}$ bimetallic catalyst with mesoporous silica nanoparticles as support and its catalysis of cinnamaldehyde hydrogenation. Journal of Catalysis, 2012, 291: 36-43

6. SR Karnati, P Agbo, L Zhang. Applications of silica nanoparticles in glass/carbon fiber-reinforced epoxy nanocomposite. Composites Communications, 2020, 17: $32-41$

7. Q Zhang, X Wang, P Li, KT Nguyen, X Wang, Z Luo, H Zhang, NS Tan, Y Zhao. Biocompatible, uniform, and redispersible mesoporous silica nanoparticles for cancer-targeted drug delivery in vivo. Advanced Functional Materials, 2014, 24: 2450-2461

8. PG Jeelani, P Mulay, R Venkat, C Ramalingam. Multifaceted application of silica nanoparticles. A review. Silicon, 2020, 12:1337-1354

9. J Che, B Luan, X Yang, L Lu, X Wang. Graft polymerization onto nano-sized $\mathrm{SiO}_{2}$ surface and its application to the modification of PBT. Materials Letters, 2005, 59: 1603-1609

10. SR Karnati, D Oldham, EH Fini, L Zhang. Surface functionalization of silica nanoparticles to enhance aging resistance of asphalt binder. Construction and Building Materials, 2019, 211: 1065-1072

11. Y Li, B Li, X Zhao, N Tian, J Zhang. Totally waterborne, nonfluorinated, mechanically robust and self-healing superhydrophobic coatings for actual anti-icing. ACS Applied Materials \& Interfaces, 2018, 10: 39391-39399.

12. M Niederberger, MH Bartl, GD Stucky. Benzyl alcohol and titanium tetrachlorides: a versatile reaction system for the nonaqueous and low-temperature preparation of crystalline and luminescent titania nanoparticles. Chemistry of Materials, 2002, 14: 4364-4370

13. W Stöber, A Fink, E Bohn. Controlled growth of monodisperse silica spheres in the micron size range. Journal of Colloid and Interface Science,
1986, 26: 62-69

14. S Cai, Y Zhang, H Zhang, H Yan, H Lv, B Jiang. Sol-gel preparation of hydrophobic silica antireflective coatings with low refractive index by base/acid two-step catalysis. ACS Applied Materials \& Interfaces, 2014, 6: 11470-11475

15. Y Li, X Men, X Zhu, B Ge, F Chu, Z Zhang. One-step spraying to fabricate nonfluorinated superhydrophobic coatings with high transparency, Journal of Materials Science, 2016, 51: 2411-2419

16. T Li, Y Ding, S Kareem, F Qiao, G Ali, C Ji, X Zhao, Y Xie. Hexamethyldisilazane-triggered room temperature synthesis of hydrophobic perovskite nanocrystals with enhanced stability for light-emitting diodes. Journal of Colloid and Interface Science, 2019, 552: 101-110

17. H Althues, J Henle, S Kaskel. Functional inorganic nanofillers for transparent polymers. Chemical Society Reviews, 2007, 36: 1454-1465 